\title{
Desarrollo de las especialidades de enfermería a 20 años de su lanzamiento por la facultad de enfermería de la Universidad Peruana Cayetano Heredia
}

\author{
Rossana Gonzales De la Cruz ${ }^{1 . a}$
}

\begin{abstract}
RESUMEN
Este trabajo busca mostrar cómo se ha construido el proceso de formación de las enfermeras especialistas de la Universidad Peruana Cayetano Heredia, pionera en los procesos de especialización en el Perú, así como los aportes que los Programas de especialización han sido utilizados para la mejora de los cuidados que se brindan en las instituciones de salud, en beneficio de los usuarios, la comunidad y también para las mismas profesionales. Para la elaboración del artículo se ha utilizado fuentes estadísticas de la universidad así como del programa de especialización en enfermería y el material bibliográfico resultante de la investigación científica publicada en los últimos veinte años,con relación al tema,y cuyo análisis del contenido se empleó para el tratamiento de los datos encontrados. Destacan como datos relevantes, que la especialización es necesaria para la formación y el desarrollo de las competencias para actuar en un mercado laboral cada vez más exigente y competitivo; las egresadas reconocen al programa por su contribución tanto al desarrollo de las competencias requeridas como especialistas, así como al mejoramiento de su posición laboral. Los retos y recomendaciones analizan la situación del mercado laboral, la formación y el contexto global, permitiendo presentar sugerencias para hacer un programa todavía y especialistas más eficientes para su continuo desarrollo profesional.
\end{abstract}

PALABRAS CLAVE: Programa académico, especialización en enfermería, competencias, retos de la enfermera especialista

\section{Development of nursing specialties 20 years after its launch by the nursing faculty at the Universidad Peruana Cayetano Heredia}

\section{SUMMARY}

This article seeks to show how he has built the formation of RNs at the Universidad Peruana Cayetano Heredia, a pioneer in the process of specialization in Peru, as well as the contributions that specialization brings training services, the user the community and to the same professional. We used statistical sources university and the program and bibliographic material resulting from scientific research published in the last twenty years, regarding the issue, and whose content analysis was used for the treatment of the data found. Stand out as relevant data, that specialization is necessary for the formation and development of the power to act in an increasingly demanding and competitive labor market; the program graduates recognized for their contribution both to the development of specialist skills required as well as the improvement of their working position. The challenges and recommendations discussed the situation in the labor market, training and global context, allowing forward suggestions for a program even more efficient and continuous professional development for specialists.

KEYWORDS: curriculum, specialization in nursing skills, challenges nurse specialist.

Hospital Edgardo Rebagliati Martins, EsSalud. Lima, Perú.

Licenciada Especialista en enfermería. 


\section{INTRODUCCIÓN}

La especialización en enfermería hoy se impone y se difunde en todos los países como consecuencia natural del progreso, de la profundidad del conocimiento en todos los sectores, y en la enfermería en particular, confirmando que se hace imposible satisfacer las demandas de salud cada vez más complejas solamente con la formación en pregrado. La especialización en enfermería enel mundo, tiene más de cincuenta años de desarrollo; en nuestro país lleva ya 17 años, actividad que se inició, en su modalidad escolarizada, en la Facultad de Enfermería (FAEN) de la Universidad Peruana Cayetano Heredia, que hoy cumple 100 años de vida institucional ininterrumpida, en su transformación de escuela a facultad, siendo líder y generadora de los programas de especialización a nivel nacional.

El presente artículo pretende mostrar el desarrollo histórico del programa, su aporte a la formación de las nuevas generaciones de enfermeras, así como su aporte a la salud y al cuidado especializado y, por ende, a la calidad y seguridad de los usuarios, familias y comunidad. Así mismo, expone el impacto del proceso formativo desde la opinión de los exalumnos y directivos; se muestran los retos y las recomendaciones basadas en el análisis de expertos en el sector Salud, en la educación y la práctica avanzada de Enfermería, con la finalidad de seguir retroalimentando el proyecto educativo que le permita alcanzar la meta social de la profesión.

La evolución de la formación de enfermeras en el Perú, transcurre como parte de la quinta etapa correspondiente a la expansión de la formación universitaria de pre grado y desarrollo de los programas de perfeccionamiento iniciada en 1991(1).Desde inicios de los 90, posterior al convenio de afiliaciónde la Escuela de Enfermeras Arzobispo Loayza con la Universidad Peruana Cayetano Heredia, se evidenció el interés en ejecutar un programa de posgrado en Enfermería (1); es entonces que en 1991 que se conformó la Comisión de Posgrado en Enfermería con la finalidad de estudiar las posibilidades de poner en marcha programas de especialización y maestría;existía evidencia que alrededor de los programas de posgrado se podrían formar escuelas de excelencia académica que mejoren la calidad del cuidado y el desarrollo del conocimiento a través de la investigación.

El proyecto de especialización buscaba aportar al desarrollo de la atención integral de salud con enfoque interdisciplinario, orientado a contribuir al desarrollo de salud del país en áreas prioritarias generando conocimientos y experiencias aplicadas a la realidad peruana integrando el servicio, la docencia y la investigación ${ }^{2}$; lo que se hace evidente en los dos ejes del currículo:Gestión e Innovación del Cuidado y Gestión e Innovación de los Servicios de Enfermería.

Las áreas de especialización consideradas fueron Enfermería en Salud Comunitaria,Enfermería Pediátrica,Enfermería en Cuidados Intensivos,Enfermería Quirúrgica,Enfermería Oncológica, Enfermería Psiquiátrica y Enfermería Geriátrica. El plan de estudios incluía contenidos de humanidades, ciencias sociales, profesionales y de especialización.

En 1997, el Departamento Académico retomó y actualizó el proyecto, el cual fue aprobado en Consejo Universitario.En 1998 la Facultad de Enfermería de la Universidad Peruana Cayetano Heredia ofreció por primera vez el Programa de Especialización bajo la modalidad escolarizada, inicialmente en las especialidades de Enfermería en Cuidados Intensivos, en Emergencias y Desastres y Enfermería Neurológica (2); de ese modo se constituyó en el primer programa, a nivel nacional, en desarrollar la especialidad escolarizada de Enfermería. Posteriormente, en el 2000, se abre el programa no escolarizado en la Universidad Nacional Mayor de San Marcos.

El programa de especialización contó con la cooperación de OPS/OMS a través del Centro Colaborador de Educación en Enfermería (ACOFAEN) de Colombia, uno de los países líderes en la formación de especialistas en el contexto latinoamericano; así mismo,en vinculación con la Facultad de Enfermería de la Universidad de Alberta Canadá se planificó y, posteriormente,se desarrolló la Especialidad de Salud Mental y Psiquiatría. En la actualidad se vienen desarrollando quince especialidades de enfermería que se inician o cierran de acuerdo al requerimiento de los profesionales de Enfermería y de los servicios de salud.

La tres primeras especialidades iniciadas en 1998 fueron: la Especialización de Enfermería en Cuidados Intensivos,Enfermería en Emergencias y Desastres y Enfermería Neurológica,las cuales contaron con el soporte de docentes internacionales de la Universidad Nacional de Colombia y de la Universidad de Antioquia,quienes motivaron a las dos primeras cortes de estudiantes del programa, con su excelencia académica y profesional. Actualmentelas dos primeras especialidades cuentan con36 programas de especialización que han formado más de 400 especialistas; en tanto, que Enfermería Neurológicacuenta con dos programas de especialización, el primero en 1998,y 
el segundo en el 2005, que se abrió por la demanda de las colegas del Instituto Neurológico Santo Toribio de Mogrovejo del Ministerio de Salud (MINSA).

La Enfermería en Salud Mental y Psiquiatría se abre en 1999 y desarrolló solo un programa ya que, al mismo tiempo,otra universidad inició la especialidad bajo la modalidad no escolarizada; no se tiene referencia de que se haya abierto otro programa similar en alguna universidad del país, a pesar de ser una de las área prioritarias del cuidado especializado.

La especialización en Enfermería Pediátrica se inició en el año 2000 y contó con la participación de una enfermeraespecialista en enfermería Pediátrica docente de la Pontificia Universidad Catolica de Chile.

La Enfermería en Centro Quirúrgico Especializado se inició, igualmente, en el año 2000, de esta especialización se realizaron 28 programas, en este caso cabe resaltar que el primer programa por convenio con la Asociación de Profesionales Enfermeros Especializados en Centros Quirúrgicos convocó a 87 miembros de la organización, pertenecientesa los hospitales del MINSA, EsSalud y de las Fuerzas Armadas.En el año 2001 se abre el programa de Cuidados Intensivos Neonatales al igual que Cuidados Intensivos Pediátricos (3), de ellos se han desarrollado programas para enfermeras de institutos y hospitales del nivel III, que cuentan con estos servicios especializados.

En el año 2005 se apertura el programa de especialización de Enfermería en Cuidados Cardiovasculares,programa que se inició en convenio con el Instituto de Salud Cardiovascular de EsSalud y que en la actualidad ya ha desarrollado diez programas;asimismo, se abren Enfermería en el Cuidado de la Mujer y el Recién Nacidoque tuvo una promoción, y Cuidado Nefrológico, que a la actualidad lleva diez programas. La especialidad de Enfermería en Cuidado Oncológico fue aprobada en el año 2008 y se han desarrollado cinco programas hasta hoy. La especialidad de Cuidados Cardiovasculares Pediátricos, abierto en el año 2012,fue posible gracias al convenio con el Instituto Nacional del Corazón (INCOR) y el INSN, el cual brindó becas de estudio a sus enfermeras; el mismo año, la especialidad de Cuidados en Gastroenterología, Endoscopía y Procedimientos Especiales inició sus actividades y hasta la fecha se han desarrolladodos programas.Salud Ocupacional es la última especialidad en abrirse en el 2014, y la primera promoción se encuentra en cursando el segundo semestre de formación.
Así mismo,el 2004 a solicitud del Consejo Regional del Colegio de Enfermeros del Perú se llevó a cabo un programa descentralizado con tres especialidades en la ciudad de Huancayo; estas fueron: Cuidados Intensivos, Emergencias y Desastres,y Centro Quirúrgico Especializado, en concordancia con los requerimientos de la región Junín(4).

En los 17 años de desarrollo del programa se han realizado evaluaciones de su pertinencia yse han formado comisiones de autoevaluación,como la del año 2013 que presentó un informe que permitió realizar los cambios al actual Programa de Especializaciónvigente, a partir del programa 39; en él se fortalece la formación en el eje disciplinar a través del curso Ciencia del Cuidado.La autoevaluación ha permitido adecuar el programa a los avances científicos y tecnológicos, a las necesidades de la sociedad, del sector, de la profesión, así como plantear nuevos programas de especialización, los cuales se encuentran en fase de planificación.

\section{Contribuciones del programa}

Las especialistas en Enfermería son enfermeras de práctica avanzada que aportan conocimiento especializado para el desarrollo de la práctica a través de tres esferas de influencia: facilitando los resultados de calidad para los usuarios; el apoyo y mentoría al personal de enfermería y liderando cambios innovadores que permitan avances en el sistema de salud, para atender las necesidades de los usuarios, las familias y las comunidades. Para esto, las especialistas emplean siete competencias básicas en su práctica: la atención directa;la consultoría,el liderazgo en el sistema de salud; de colaboración en el equipo multidisciplinario; de capacitación; de investigación, y de toma de decisiones éticas.

Es así que a partir del año 2000 que egresa la primera promoción de especialistas de la FAEN se hace evidente la contribución del Programa y sus especialistas, tanto a los servicios como a la formación de especialistas del contexto nacional. Diversos hospitales y clínicas del país envían a formar a sus profesionales de enfermería para poder abrir servicios de emergencia, cuidados intensivos de adultos y pediátricos en distintos puntos del país y, de esta manera, poder organizar los nuevos servicios y cumplir con los criterios de acreditación en sus respectivas instituciones. Para otros servicios ya constituidos y en actividad, les permite cumplir con los estándares de acreditación nacional e internacional requeridos, como sucede en el INSN; el INCOR; el Instituto Nacional de Ciencias Neurológicas; 
los hospitales nacionales Arzobispo Loayza, Cayetano Heredia, Edgardo Rebagliati, Alberto Sabogal y de Emergencias Pediátricas entre otros.

En cuanto al aporte que realiza el Programa de Especialización al fortalecimiento de los servicios de salud y de enfermería,este se realiza a través del convenio docente asistencial con nuestras sedes de práctica (5) en las cuales docentes y estudiantes de la especialidad participan activamente en los programas de educación continua y cursos de actualización para las profesionales y personal técnico en temas concernientes a la especialidad;así mismo, participan en actividades de mejoramiento continuo como el desarrollo de guías de cuidado, nuevos registros de Enfermería y el desarrollo de proyectos de investigación-acción que permiten contribuir al mejoramiento continuo de la calidad del cuidado en la especialidad.

Este aporte se hace extensivo a todos los hospitales e instituciones de salud de donde provienen nuestros estudiantes; en este compromiso asumido con nuestros egresados, brindamos asesoría y consultoría para la implementación de nuevas herramientas y tecnologías aplicadas al cuidado especializado (por ejemplo: ventiladores de alta frecuencia, nuevas modalidades ventilatorias en cuidados intensivos neonatales) participando, además, en cursos y congresos de las especialidades desarrollados tanto en el entorno nacional como internacional.

El aporte al cuidado es realizado a través de la constante actualización de conocimientos, e incorporación de la evidencia científica al desarrollo de la actividad docente y su implementación en la práctica de la especialidad; tal es así que en estos 17 años hemos sido líderes en el desarrollo e implementación del proceso de cuidado de enfermería así como en el desarrollo de registros de valoración y cuidado en el área de cuidados intensivos, en la actualidad nos encontramos utilizando las taxonomías NANDA, NIC y NOC, para el desarrollo del plan de cuidado de la especialidad. Igualmente, se aportó al desarrollo del compendio de guías de intervenciones y procedimientos de enfermería para casos de desastre (5).

A partir de nuestras docentes y egresadas de nuestros programas de especialización se han generado propuestas innovadoras para el cuidado especializado como la implementación de programas de mejoramiento continuo de la calidad y seguridaden los servicios, humanización del cuidado al paciente y la familia (6) prevención de infecciones así como la Jornada por el Día de Salud Renal,entre otros.
En cuanto a la contribución académica en la formación de especialistas a nivel nacional, las alumnas egresadas de los primeros programas se constituyeron en los primeros docentes con formación escolarizada de nuestra propia casa de estudio, así como de programas de especialización escolarizada de diferentes universidades a nivel nacional; se menciona a algunas de ellas como la Universidad Nacional Mayor de San Marcos, Universidad Peruana Unión, Universidad Nacional de Trujillo, Universidad Nacional San Luis de Gonzaga, Universidad San Antonio de Abad, Universidad del Altiplano, Universidad de San Martín de Porres, Universidad NorbertWiener, Universidad Garcilaso de la Vega, entre otras. Por otro lado, la Facultad consideró conveniente autorizar asesorar la creación de nuevos programas de especialización en el interior del país, considerandolas necesidades y prioridades locales y regionales, ejemplo de ello es el proyecto de creación del Programa de Especialización de la Universidad Nacional de San Antonio Abad del Cusco. En estos programas las especialistas se constituyen en efectivo personal de soporte a la docencia ya sea como docentes y/o tutoras de práctica donde hacen evidente su liderazgo y expertísimo en la atención de salud y el cuidado especializado de enfermería.

Otro aporte importante como producto de los programas de especialización de nuestra casa de estudios ha sido la generación, desde la formación, de espacios de reflexión, compromiso y liderazgo profesional lo cual llevó a la conformación y fortalecimiento de sociedades de enfermería de las principales especialidades, entre ellas: la Sociedad Peruana de Enfermeras Especialistas en Cuidado Critico (SOPECC), Sociedad Peruana de Enfermeras Especialistas en Emergencias y Desastres (SOPED), la Sociedad Nacional de Enfermeras Materno Infantil, pero no solo se conforman sociedades científicas, sino que se desarrollan actividades de actualización en la especialidad,así mismo, son parte del proceso de certificación de especialistas, en trabajo conjunto con el Colegio de Enfermeros del Perú.

El liderazgo demostrado por las especialistas ha trascendido los límites nacionales, llevando a tales organizaciones a formar parte de redes internacionales como la Federación Mundial de Enfermeras de Cuidado Crítico (WFCCN) (7) de la que son parte la SOPECC y la SOPEED; así mismo, llevó a generar redes latinoamericanas como la Federación Latinoamericana de Enfermeros de Cuidados Intensivos,de cuya organización las enfermeras peruanas fueron líderes y artífices en conjunción con los líderes de la WFCCN quienes asesoraron estos procesos de creación y desarrollo, siendo los dos primeros periodos de gestión de responsabilidad del Perú $(8,9)$;en los que se difundióy 
fortaleció el conocimiento y las prácticas de la especialidad en el contexto latinoamericano.

Como se puede apreciar, las especialistas han puesto en práctica sus conocimientos y competencias tanto en el cuidado especializado como en la organización de servicios y el desarrollo de organizaciones científicas de enfermería haciendo evidente la formación integral recibida, así como el compromiso y liderazgo personal asumido con la especialidad.

En la investigación titulada "Percepción de la formación desde la perspectiva de las especialistas" (10)se encontró que $76 \%$ de las egresadas de la FAEN se halla ejerciendo como especialista; el 18\% como coordinadora de enfermería; el 11\% como enfermera jefe, y el 16\% como docente,pero el $24 \%$ aún se encuentra en otras áreas como enfermeras generales, lo que evidencia la subutilización del recurso en los servicios de salud (10).

Con relación al perfil ocupacional,más del $70 \%$ de las egresadas consideran que los estudios de especialización aportaron al logro de las principales competencias referidas a la evaluación, provisión de cuidado del paciente y de la familia, así como para la aplicación de herramientas para el cuidado y la gestión del mismo. Así mismo, el 63\% considera que la especialización ha contribuido a mejorar su posición laboral dentro de la institución.Lo que hace evidente la contribución del programa al desempeño y posicionamiento laboral del programa de especialización.

En cuanto a la evaluación de los programas, las ex directoras del Programa de Especialización de la facultad expresaron que se han generado cambios en la estructura del programa,fortaleciéndolo para ofrecerlo no solo en Lima, sino en forma descentralizada incrementando el acceso y desarrollo de las profesionales de enfermería de las regiones, lo que a su vez a consolidado a las instituciones de salud a través de los servicios que ofrecen las enfermeras especialistas, las cuales se constituyen en educadoras, consultoras y líderes en la atención de salud y el cuidado especializado; este el más alto logro de la productividad social de la universidad.Así mismo, consideran importante el trabajo colaborativo entre las instituciones formadoras y la creación y fortalecimiento de las sociedades de especialistas de enfermería, pues contribuyen al desarrollo legal, científico y académico de la Enfermería, constituyéndose en un nuevo desafío el trabajo colaborativo en redes nacionales e internacionales.

\section{De los retos y desafios}

La preparación de una fuerza laboral sólida conlleva muchos desafíos por lo que el Programa de Especialidad en Enfermería se constituye en una instancia de formación de avanzada que brinda capacidad de respuesta a las nuevas demandas sociales y a los cambiantes requerimientos educativos. Así mismo, en una época donde la ciencia y la tecnología revolucionan todos los espacios de la vida del ser humano, la Enfermería no es la excepción, esta ha sentido el impacto de dichos avances, a la vez que ha participado en ellos, lo cual la ha comprometidoa mantenerse actualizada, consumir y producir conocimientos, sinperder de vista que su compromiso fundamental es con las personas en una relación de respeto y solidaridad. Por lo tanto, pensar en los retos específicos que supone el actual escenario de la formación y el ejercicio de la especialidad en el entorno nacional y global,nos lleva a reflexionar en elementos claves, como por ejemplo: los retos propios al proceso de Reforma del Sector Salud (11),que tiene como primer reto: Extender mejoras en el estado de salud a toda la población,lo cual requiere incentivar desde la formación el cuidado con visión multidimensional e integral, así como abrir el paradigma de acción de la Enfermería como responsable del cuidado de la salud y calidad de vida de las personas, hacia un trabajo y posicionamiento multisectorial. La propuesta de formación de Enfermería debe incluir un proceso fuera de lo convencional que abarque el diseño de nuevos perfiles ocupacionales teniendo en cuenta los aportes de los usuarios,para el logro de los cambios en la calidad deseado,transformando la relación de la enfermería consigo misma y con la sociedad; en este sentido, la FAEN se preocupa por ofertar especialidades que se ajusten a las necesidades del sector y de la población, teniendo en cuenta sus requerimientos.

El segundo reto es: Instaurar cultura de prevención y protección de la salud en la sociedad, lo cual requiere del fortalecimiento y trabajo intenso en la generación de competencias para la asesoría,consejería y educación para la salud, tanto innovadoras en sus recursos y estrategias como las basadas en mejor evidencia científica que permita lograr el impacto requerido para la trasformación de los estilos de vida de nuestra población, así como el fortalecimiento del primer nivel de atención, con la participación proactiva de las enfermeras especialistas,por lo que el programa debe recrear una especialidad comprometida con el cambio de su práctica asistencial, construyendo las transformaciones sobre la base de reflexiones sólidas, con apertura y disposición para trabajar con innovaciones apropiadas al quehacer profesional de la práctica de salud 
y la enfermería,lo que requiere del enriquecimiento de las relaciones recíprocas de participación y aclaración de los roles y procesos de trabajo, incluyéndose el ser educadores, administradores de casos, líderes de proyectos de mejora e investigadores.

El informe publicado por The Institute of Medicine (IOM) en colaboración con la Fundación Robert Wood Johnson, sobre el futuro de la Enfermería (12), fue tomado como guía en los Estados Unidos para establecer las metas y objetivos de la profesión de Enfermería para la próxima década. Con la meta de asegurar que la Enfermería esté preparada para asumir roles de liderazgo, apuntando a mejorar el cuidado del paciente y los sistemas de salud; este informe identificó cuatro mensajes clave y ocho recomendaciones, de las cuales cabe resaltar:

Las enfermeras deben practicar con todo el peso de su educación y formación para la protección de la salud y seguridad de los pacientes mediante la reglamentación adecuada de la formación y de la práctica de enfermería (13),lo que exige una reglamentación y regulación concertada de organizaciones, en nuestro caso el Colegio de Enfermeros, la Asociación Peruana de Facultades y Escuelas de Enfermería y las sociedades científicas, que garantice la calidad de especialistas del país; la FAEN, comprometida con la calidad educativa, realiza la autoevaluación del programa camino al proceso de normalización de los programas de especialización de Enfermería.

Las enfermeras deben lograr mayores niveles de educación y formación a través de un sistema de educación que promueve la progresión académica sin fisuras(OIM 2011),para lo cual los programas de especialización deben estar articulados con los programas de maestría clínica y doctorado en la práctica de enfermería, de manera que se permita la progresión y consolidación de la práctica avanzada de Enfermería, acorde con los avances de la Enfermería mundial y que, a su vez, le permitan el desarrollo de productos científicos que fortalezcan el cuidado especializado, la salud y la profesión; en tal sentido, los posgrados de la FAEN están articulados de manera que permitan la progresión del profesional de Enfermería, siendo uno de sus retos la oferta de estos nuevos posgrados para la Enfermería nacional.

Así mismo, se requiere de profesores de Enfermería que con estos niveles de formación avanzada consoliden el desarrollo de estos programas de posgrado y el liderazgo de las profesionales de práctica avanzada.
Garantizar el compromiso del enfermero con la capacitación permanente, por lo que es fundamental,desde la formación,animar y gestionar la progresión del aprendizaje a través de programas de educación continua (14) que permitan la certificación y renovación de sus capacidades profesionales en el continuo de formación establecido por el Consejo Internacional de Enfermería (CIE).

Las enfermeras deben ser socias de pleno derecho, con los médicos y otros profesionales de la salud, en el rediseño de la atención de salud nacional y global,para lo cual en la formación de Enfermería no solo deben incluir habilidades de liderazgo y gerencia,sino quese debe tomar mayor responsabilidad para tutelar y desarrollar a los líderes, por lo cual, en los programas de especialización se debe incentivar y fortalecer la capacidad de liderazgo, gerenciamiento y desarrollo político.

La formación para la promoción de políticas, diseño y gestión de planes de desarrollo le permitirán su participación en los diferentes niveles de decisión del sector y la política del contexto nacional y global.

En nuestro país, la formación de las especialistas de Enfermería está sujeta a la LeyUniversitaria 30220 ya la Ley del Enfermero Peruano 27669, que en su Capítulo Vartículo 16 reconoce los estudios de especialización (15); así mismo, en la reforma del sectorse ha reconocido estructural y económicamente, después de 17 años de existencia, por el Ministerio de Salud, pero no siempre estos profesionales son absorbidos como tal por los servicios de salud, como lo demuestran los resultados de la investigación realizada por la SOPECC; teniendo en cuenta que el trabajo que ejercen en la práctica asistencial no los distingue de los no especialistas; estos profesionales dejan de ser reconocidos como tal por su ubicación irregular y todista dentro de los servicios de salud, así como por la ausencia de un plan de desarrollo estimulado por la educación continua, su propio interés y el de la gestión del talento humano de la institución de la que es parte.

Articular el trabajo del Colegio Profesional, de entidades formadoras, sociedades científicas y entes reguladores, es una prioridad para asegurar la calidad de los recursos humanos de Enfermería y su correspondencia a las necesidades del país.La regulación debe implementarse obligatoriamente en los terrenos académico y profesional, contribuyendo así al más elevado estado profesional de las enfermeras basado en los principios y valores universales consagrados en el Código Deontológico del CIE(16). 
Otro reto en el proceso de formación del estudiante siglo XXI es la diversidad del nivel de formación básica (17),diversidad cultural y manejo de tecnología, que requiere que el programa a través del currículo y el desarrollo de estrategias específicas busque llenar las brechas para el logro de las competencias del futuro especialista.Internacionalización en la formación de profesionales de Enfermería, en el marco de la globalización de los servicios de salud que permita el libre movimiento de profesionales en el orbe, requiere incorporar nuevos paradigmas y estrategias diversas para este fin.

Con relación a las innovaciones metodológicas, la evidencia científica recomienda el método de simulación: En Instituciones de educación y formación de profesionales de la salud se deben utilizar simulación de altos niveles de fidelidad, contextualmente apropiadas en la educación de los profesionales de la salud.La calidad de la evidencia que apoya estas recomendaciones es moderada, y la fuerza de la recomendación es fuerte (18). Organizaciones como la ANA, ACCN y la Agencia Para la Investigación de la Calidad apoyan y estimulan esta innovación que crea mayor aprendizaje y cumple un rol muy importante en la mejora de la seguridad y la gestión de riesgos (19). Entre los recursos requeridos para fortalecer el proceso educativo y el desarrollo de las competencias de las especialistas se incluye el contar con aulas de simulación de alta fidelidad con apoyo informático que facilite el logro de la competencia, acorde con lo mencionado previamente.

Los campos clínicos, actualmente abarrotados y con pocas oportunidades, se constituyen en una barrera para el logro de competencias prácticas y la integración de competencias teóricas a la práctica,por lo que son las aulas de simulación un entorno que facilitaría el logro de las competencias.

Por otro lado, el cuidado con el apoyo de las redes digitales interoperables (HIT) desplazará la importancia del tiempo y el lugar.El paciente no necesita estar en la misma ubicación que el proveedor, y este no siempre necesita interactuar con el paciente en tiempo real. Existe una gran variedad creciente de dispositivos biométricos (por ej. monitores cardiacos o de glucosa en sangre permanentes) que pueden recoger, monitorear y reportar la información del paciente en tiempo real, ya sea en una institución o en el hogar.Algunos de estos dispositivos también pueden proporcionar terapias, como la bomba de insulina, así como los desfibriladores implantables. No está claro qué parte de la atención de enfermería podría ser "geográficamente sin ataduras" cuando HIT se aplique plenamente, pero es probable que sea un subconjunto significativo de la atención, en el rango de 15 a $35 \%$ de lo que las enfermeras hacen hoy; requiriendo del cambio de la visión de la relación enfermera-paciente y el contexto del cuidado, lo que también exige cambios en la formación.

\section{RECOMENDACIONES}

La educación de Enfermería debe convertirse en un completo socio en el rediseño del sistema de salud a través de su participación en iniciativas de rediseño y modernización para satisfacer las necesidades de servicio(20). Una colaboración significativa entre la educación de Enfermería y rediseño de la prestación de asistencia sanitaria fomentará la alineación en sus objetivos, que es fundamental para el éxito conjunto.

A los docentes les cabe el papel de estimuladores del proceso educativo (21),promoviendo la libertad,siendo copartícipes del proceso de construcción histórico-social en el cual, el futuro especialista es responsable por su proceso permanente de calificación y formación, a decir de Asmann (1998) el conocimiento se transformó en asunto obligatorio y volvió a ejercer fascinación en esta sociedad del conocimiento, afirmando:Es preciso substituir la pedagogía de las certezas y de los saberes prefijados por una pedagogía de la pregunta y del acceso a las información(...), por una pedagogía de la complejidad que sepa trabajar con conceptos versátiles abiertos a lo imprevisto (21).

El desarrollo de competencias debe pasar de competencias basadas en tareas a las competencias de alto nivel,guiando a los estudiantes en la integración de los conocimientos de las ciencias clínicas, sociales y de comportamiento, con la práctica de la Enfermería para mejorar el desarrollo de habilidades de razonamiento clínico.Además de la generación de nuevas competencias para la toma de decisiones, la mejora de la calidad, el pensamiento sistémico y el liderazgo del equipo.

Las Especialistas en Enfermería pueden, y deben,mejorar los resultados del paciente mediante la promoción de EBP;para ello, tienen que trabajar en contextos de integración docencia-asistencia y en proyectos de mejoramiento calidad y seguridad del paciente a decir de Berwick, Cronenwett:Liderando equipos que trabajan para mejorar la atención de salud (22).

Contar con un plan de desarrollo para los docentes en 
Enfermería que permita contar con los modelos y recursos idóneos, que cuenten con maestrías clínicas y doctorado en la práctica de Enfermería, para guiar y generar el desarrollo de las nuevas generaciones de especialistas.

Lograr una convivencia cooperativa y productiva entre la enfermera generalista y la especialista, así como entre las distintas especialidades y otras profesiones de la salud, también está en el listado de tareas pendientes difíciles, pero necesarias de realizar.

Sugiero se pueda incluir el reto de usar y producir conocimientos para el cuidado especializado.

\section{CONCLUSIONES}

Puede decirse que las especialidades en Enfermería vienen a suponer el mayor avance de la práctica enfermera de las últimas décadas. El incremento y profundización de las competencias de la enfermera especialista es trascendente en la calidad de los cuidados enfermeros que se otorguen a la población.Las especialidades de Enfermería implican el rediseño de la organización del cuidado y de los servicios de enfermería, por lo que es necesario mostrar mayor evidencia que los ubique en el lugar que les corresponde dentro de los servicios sanitarios. Aunque es difícil de probar la causalidad, la literatura sugiere que la calidad de la atención depende en gran medida de las enfermeras. El estado futuro de la Enfermería está ligada a los avances en la calidad de la atención al paciente y la seguridad que son críticos para el éxito del sistema de salud de Estados Unidos hoy y mañana (Joint Commission, 2010). Si bién las medidas de calidad se han centrado históricamente en condiciones o enfermedades, muchas de las medidas de calidad utilizados en los últimos años abordan lo bien que las enfermeras son capaces de hacer su trabajo (Kurtzman y Buerhaus, 2008) siendo esta una oportunidad para mostrar el impacto de las especialistas en el cuidado,los servicios y la salud de las personas (23).

La superación del techo del desarrollo enfermero del área clínica a nivel nacional, hasta fines de los noventa, sirvió como reconocimiento y aliciente a profesionales en cierta medida sumidas durante décadas en la rutina y falta de actualización llevándolas al empoderamiento del conocimiento especializado.Las especializaciones de Enfermería, tras 17 años de evolución, deben mantenerse ancladas en la realidad de la asistencia de salud, protagonizada y desarrollada por las especialistas, teniendo como norte el proyecto pedagógico enmarcado en la visión filosófica,solo así se podrá conquistar la deseada transformación,que fundamente el reconocimiento de que el cambio es posible en un proceso crítico-creativo continuo de construcción y reconstrucción.

Retos e incertidumbres se presentan frecuentemente en nuestro escenario laboral, uno de los principales es que las enfermeras creamos en el significado y la trascendencia de las especialidades y las pongamos en valor, lo cual significa aplicarlas, desarrollarlas, innovarlas y utilizarlas con rigor. Es necesario que los responsables políticos y los gestores del sector salud potencien las especialidades enfermeras y las utilicen como elemento para mejorar el sistema sanitario y asegurar la cobertura universal de salud.

\section{REFERENCIAS BIBLIOGRÁFICAS}

1. Zárate M.De Escuela a Facultad transiciones en la Educación de la Enfermería en el Perú. Lima, Perú: Editorial de la Universidad Peruana Cayetano Heredia. 2013.

2. Edilma M. Suárez C. Políticas de Salud en los modelos de desarrollo. Investigación en Enfermería: Imagen y Desarrollo. 2003;5(1-2):29-37.

3. Medina L. Construcción de una definición de enfermería clínica desde una perspectiva investigativa. Investigación en Enfermería: Imagen y Desarrollo. 2005; 7(1-2):14-21.

4. De Sena R, Duran M.La Especialización Estratégica para la excelencia de la práctica. Recurso de Enfermería. Contribuciones al Proceso de Desarrollo. Bogotá: Editorial Unibiblos; 2000. p. 96-98.

5. Kleinpell R, Scanlon A, Hibbert D, Ganz F, East L, Fraser D, Wong F, Beauchesne M. Addressing Issues Impacting Advanced Nursing Practice Worldwide. OJIN: The Online Journal of Issues in Nursing. 2014; (19)2:5.

6. Gonzales R. Informe de Campo Clínico $2{ }^{\circ} \mathrm{C}$ : Guia Cuidado de la Familia del Paciente en Condición Crítica. Lima: Hospital E. Rebagliati, Essalud; 2012.

7. AlbarránJ. Latin-American Federation of IntensiveCare Nurses (FLECI) is established. BACCNNews. 2007(Citado el 10 febrero 2015); 12(12): 108. Disponible en: http://www.readcube.com/articles/10.1111\%2Fj.14785153.2007.00212_3.x?r3_referer $=$ wol\&tracking action=preview_click\&show_checkout $=1$

8. World Federation Critical Care Nurses. AccomplishmentReport 2005-2007. Pasig City, Philippines: World Federation Critical Care Nurses;2008. (Citado el 1 febrero 2015) disponible en: http://wfccn.org/wp-content/ uploads/report_07.pdf

9. World Federation Critical Care Nurses. AccomplishmentReport 2007-2009. Pasig City, Philippines:World Federation Critical Care Nurses;2010(Citado el 1 febrero 2015) Disponible en: http:// wfccn.org/wp-content/uploads/report_09.pdf

10. Gonzales R, Cuba N. Percepción de la Formación desde 
la perspectiva de las especialistas. Lima:ÑSociedad Peruana Enfermeras Especialistas Cuidado Crítico; 2014.

11. Consejo Nacional de Salud.Lineamientos y medidas de reforma del sector salud.Lima: Consejo Nacional de salud, Ministerio de Salud; julio2013. p.8-12.

12. SochalskiJ, Weiner J.Health Care System Reform and the Nursing Workforce: Matching Nursing Practice and Skills to Future Needs, Not Past Demand. The National Academies of Sciences, Engineering, and Medicine; 2011.

13. CronenwettL. The Future of Nursing: Leading Change, Advancing Health. Institute of Medicine of the National Academes; 2011. p. 447-492. (Citado el 15 enero 2015) Disponible en: http://books.nap.edu/openbook.php?record $\mathrm{id}=12956$ \&page $=\mathrm{R} 14$

14. Cavalcanti G, Viana L, Garcia I. Las especialidades y los vínculos con la formación continua del enfermero: repercusiones para la actuación en el municipio de Rio de Janeiro. Enferm Glob. 2010; 19: 1-12

15. Congreso de la Republica. Ley del Enfermero del Perú $\mathrm{N}^{\circ}$ 23536. Lima: Diario Oficial El Peruano; 1982

16. Consejo Internacional de Enfermeras .Declaración del Consejo Internacional de Enfermeras sobre la formación y la práctica profesional de los recursos humanos de enfermería en los países de habla española de América Latina y del Caribe. La Habana, Cuba: Foro de los Recursos Humanos de Enfermería; 24 y 25 de abril de 2014.

17. Foster J, Flandes S. Challenges in CNS Practice and
Education" OJIN.2014; 19 (2).

18. Organización Mundial de la Salud 2014.Educación Transformadora para Profesionales de la Salud. Geneva: Organización Mundial de la Salud ; 2014.

19. DawnaL,NedM.El uso del entrenamiento de Simulación en la Unidad de Cuidados Intensivos.Enfermería en Cuidados críticos. 2010; 33 (1): 44-51.

20. Organización Panamericana de la Salud. Orientaciones para la Educación Inicial de Enfermería hacia el 2020. Washington DC: Organización Panamericana de la Salud OPS/OMS, Área de Fortalecimiento de Sistemas de Salud, Unidad de Recursos Humanos para la Salud. 2007.

21. Argote A, Canaval G,Fontoura M, et al. Nuevos Caminos para la especialización en enfermería:-Contribuciones al Proceso de Desarrollo. Bogota: EditorialUnibiblos; 2000. p. $120-121$.

22. Campbell D, Profetto-McGrath J. Habilidades y Atributos necesarios por especialistas en enfermería clínica para promover la práctica basada en la evidencia. Clínica Especialista en Enfermería. 2013; 27(5): 245-254.

23. Larson E.Achieving Nursing's Goals: toward the next 70 years. Revesc enferm USP. 2012(citado el 10 enero 2015); 46(3): 533-534. Disponible en :http://dx.doi.org/10.1590/ S0080-62342012000300001

Fecha de Recepción: 25 de febrero del 2015.

Fecha de aceptación: 17 de junio del 2015. 